\title{
Numerical Investigation on Chemical Equilibrium Slip Flow of High Altitude and Large Mach Number
}

\author{
Song Limin ${ }^{\mathrm{a},{ }^{*}, \text { Li Changdong }}{ }^{\mathrm{b}}$, Luo Kaic ${ }^{\mathrm{C}}$, Li Song ${ }^{\mathrm{d}}$ and Li yuankai \\ Aviation University of Air Force, Changchun, Jilin, China \\ aliminsong_2001@163.com, \\ blichangdong@126.com, 'luokai@126.com, ${ }^{\mathrm{l}}$ lisong4885@126.com, ${ }^{\mathrm{e}}$ liyuankai@163.com
}

Keywords: hypersonic slip flow; chemical-equilibrium; computational model

\begin{abstract}
In the flight of hypersonic near space vehicle from $60 \mathrm{~km}$ up to $100 \mathrm{~km}$ high, the influence of real gas effect, rarefied gas effect, and Mach number effect cannot be simulated completely by the ground test. In this paper, the selection of computational model is introduced, and then carried out the numerical research on slip flow field characteristics coupling with chemical-equilibrium effect under large Mach number of high altitude.
\end{abstract}

\section{Introduction}

In hypersonic flow, the hypothesis of calorically perfect gas is invalid, the gas molecular may dissociate and ionize, so chemical reaction must be considered for accurate solution. If the chemical reaction rate is high, gas molecular collision is sufficient, the eigenvalue time of chemical reaction is much shorter than that of flow course, and then we can consider using chemical-equilibrium state in which the hypersonic near space flight vehicle usually spending amounts of time gliding. On the condition of high altitude and large Mach number, the influence of real gas effect, rarefied gas effect and Mach number effect cannot be simulated completely by the ground test, so we can appropriately use the computational model of chemical-equilibrium hypersonic slip flow in large density regime below $50 \mathrm{~km}$ high[1] as well as higher altitude lower density regime to accurately simulate the flow condition relatively.

\section{Computational Model}

Non-slip boundary condition has to be replaced by slip boundary condition in slip regime. N-S equation with slip boundary condition is generally adopted recently which can get satisfied accurate prediction while the computational cost is less expensive[2]. We developed a general 3D, parallel, structured, finite-volume CFD code based on 3D N-S governing equations, using M-AUSMPW+ scheme and LU-SGS implicit time integration scheme[3]. We adopt the improved Maxwell slip model that take into account surface curvature, the expression of slip velocity adds one part that indicates the tangential change of normal velocity, namely $u_{s}=\frac{2-\sigma}{\sigma} \lambda\left(\frac{d u}{d y}+\frac{d v}{d x}\right)$. The improved Maxwell slip model is relatively accurate and efficient, showing better applicability in hypersonic slip flow.

The hypersonic slip flow heat flux model $q_{w}=k\left(\frac{\partial T}{\partial y}\right)_{w}+\mu u_{w}\left(\frac{\partial u}{\partial y}\right)_{w}$ is used here, this improved heat flux model in some sense improves the numerical results[4]. The accommodation coefficients have little impact on surface heat flux convergence which can be obtained using 1 2 grid Renold number in slip regime.

Use the method of curve approximation based on the characteristic data of thermo aerodynamic to fit thermodynamic relation for flow field computation. As for the parameter fit of chemical-equilibrium thermo aerodynamic properties, we usually adopt Tannehill's method [5]. 


\section{Numerical Simulation}

Chose two-dimensional cylinder with diameter of $0.3048 \mathrm{~m}$ for simulation, which is the typical configuration for hypersonic flight vehicle. The inflow is air, Ma is 25, and surface temperature is $300 \mathrm{k}$, with the assumption of completely diffuse reflection. We consider the altitude of $60 \mathrm{~km}, 70 \mathrm{~km}$, $80 \mathrm{~km}$ and $90 \mathrm{~km}$, of which the atmospheric condition is consulted by 1976 US atmospheric standard. We can see that the flow is in the hypersonic slip regime. The computational grid is shown in figure 1.

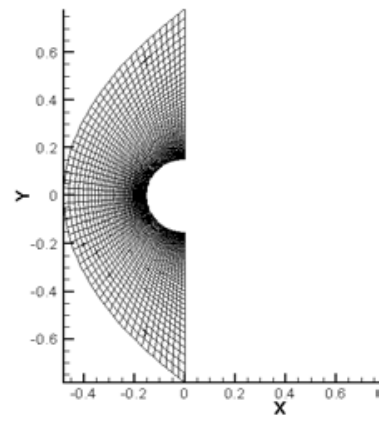

Fig.1. computational grid of tow-dimensional cylinder

Review the flow field properties such as pressure and temperature, which is non-dimensional through incoming flow and "Slip" denotes pure slip while "ChemicalSlip" is coupling with chemical-equilibrium.
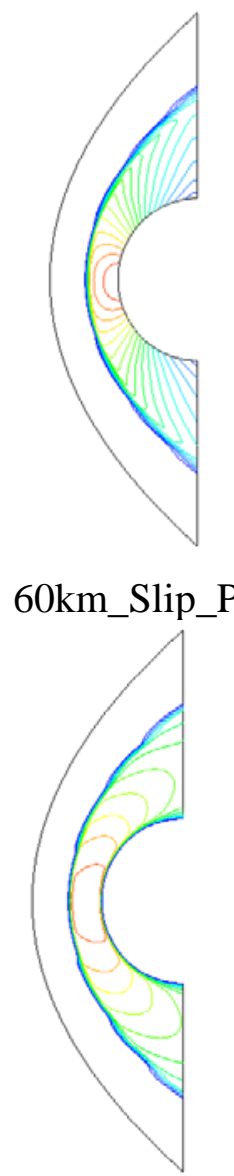

60km_Slip_T
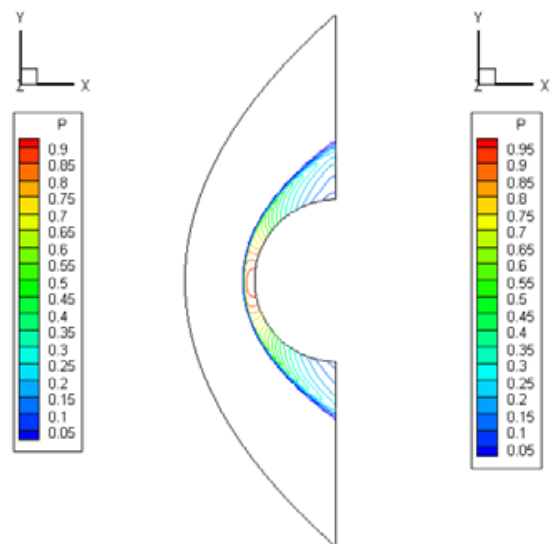

60km_ChemicalSlip_P
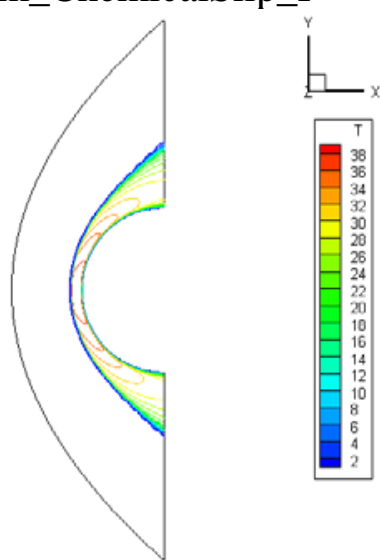

60km_ChemicalSlip_T

Fig.2. the comparison of pressure and temperature in $60 \mathrm{~km}$ 


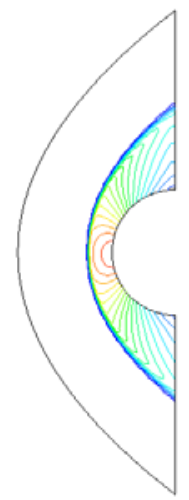

70km_Slip_P

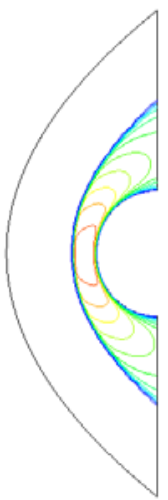

70km_Slip_T
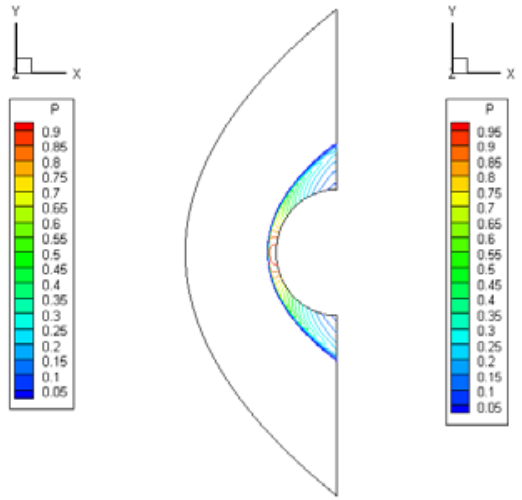

70km_ChemicalSlip_P
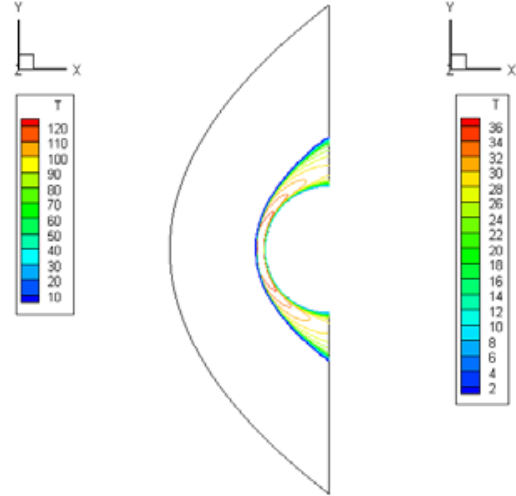

70km_ChemicalSlip_T

Fig.3. the comparison of pressure and temperature in $70 \mathrm{~km}$

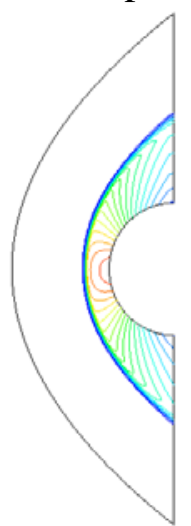

80km_Slip_P

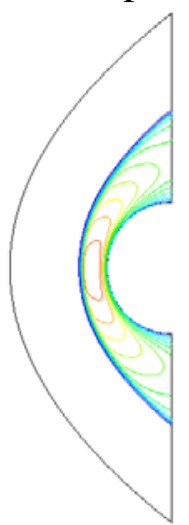

80km_Slip_T
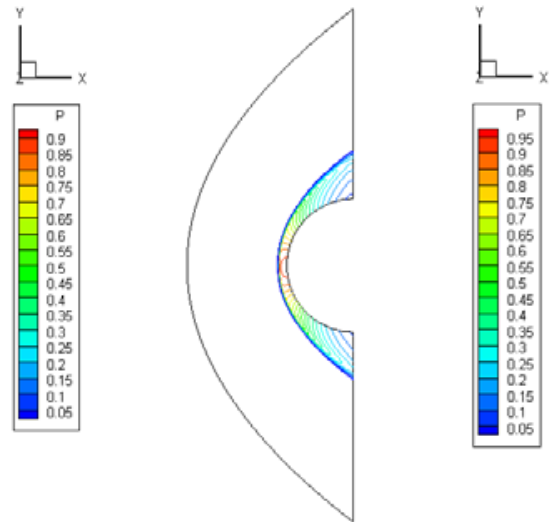

80km_ChemicalSlip_P
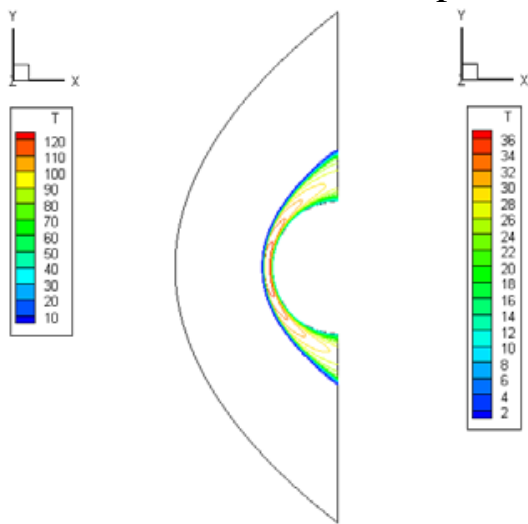

80km_ChemicalSlip_T

Fig.4. the comparison of pressure and temperature in $80 \mathrm{~km}$ 
We can see from figure 2 to 4 that considering the chemical-equilibrium effect, the shock layer becomes thinner, the pressure higher and temperature lower behind the shock wave.

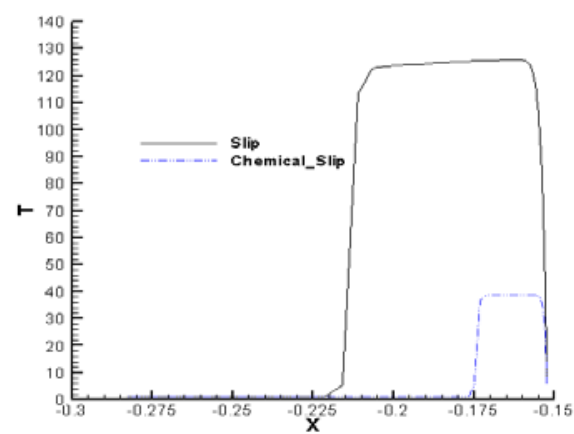

$60 \mathrm{~km}$

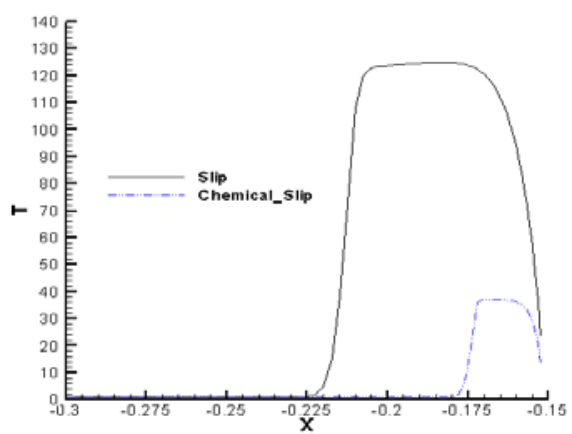

$80 \mathrm{~km}$

Fig.5. the comparison of temperature on stagnation line

Figure 5 shows the comparison of temperature on stagnation line. The y-axis $\mathrm{T}$ denotes temperature which is non-dimensional from inflow temperature. Horizontal abscissa is length with the unit $\mathrm{m}$. We can conclude that considering the chemical-equilibrium effect, the shock layer is thinner, the temperature gradient is steeper and the peak value behind shock is lower.

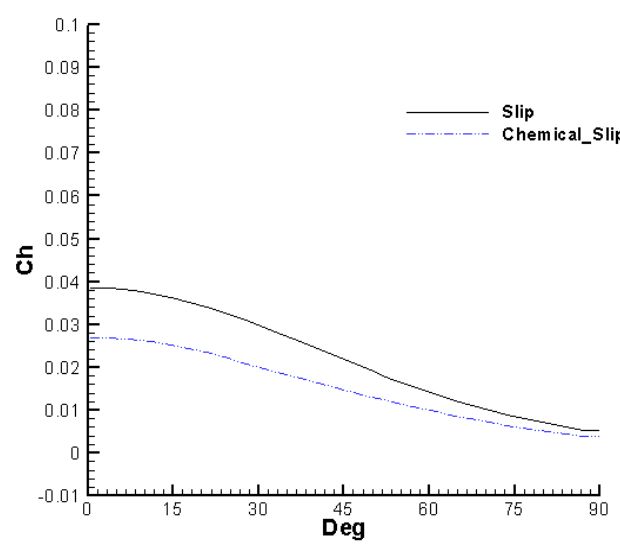

$60 \mathrm{~km}$

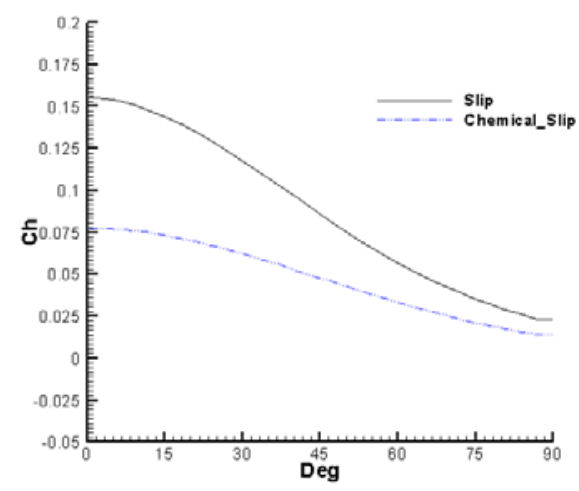

$80 \mathrm{~km}$

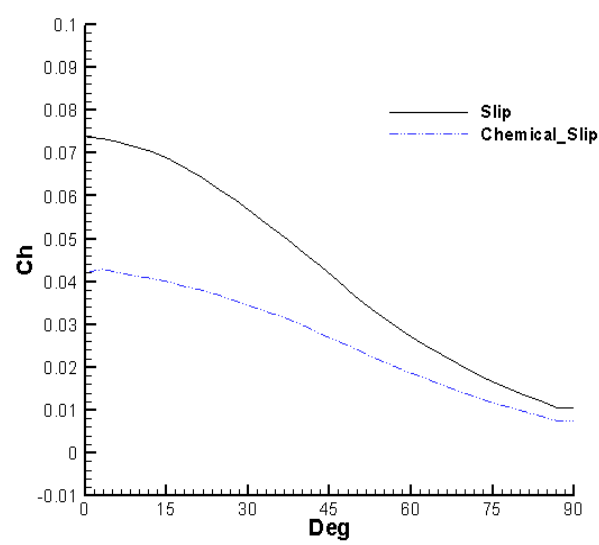

$70 \mathrm{~km}$

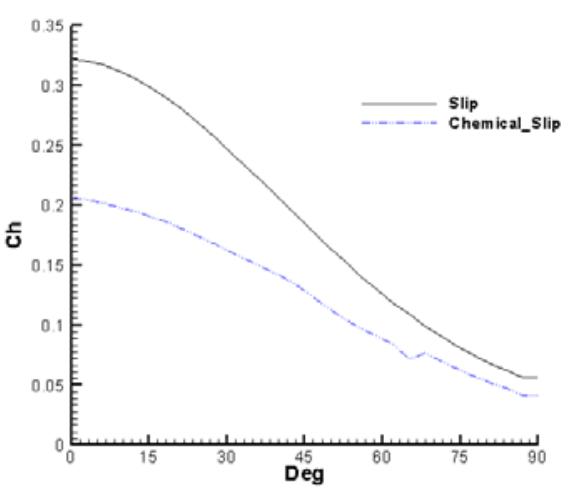

$90 \mathrm{~km}$

Fig.6. the comparison of heat flux coefficient

We analyzed the properties of flow field above, now focus on the surface properties. Take heat flux coefficient for example, the y-axis $c h=q / 0.5 \rho_{\infty} u_{\infty}{ }^{2}$, x-axis is angle which starts deasil from stagnation point(0 degree).

From figure 6 we can see that the slip flow surface heat flux coefficient comes out descending considering the chemical-equilibrium effect, at the same time, the value of ch increases with the growing on rarefied gas effect which can be demarcated by Kn number ${ }^{[2]}$. 

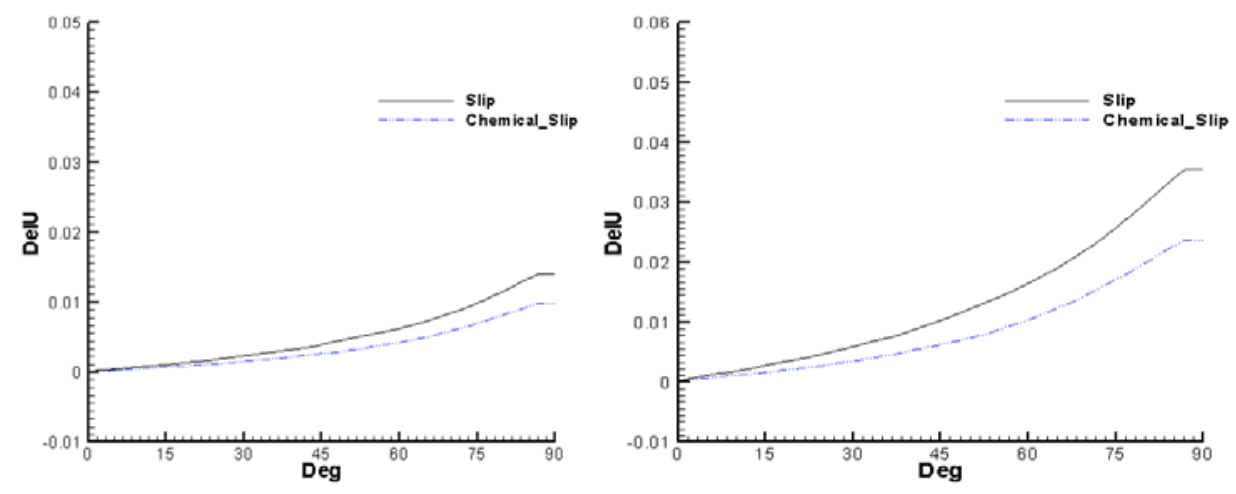

$60 \mathrm{~km}$

$70 \mathrm{~km}$
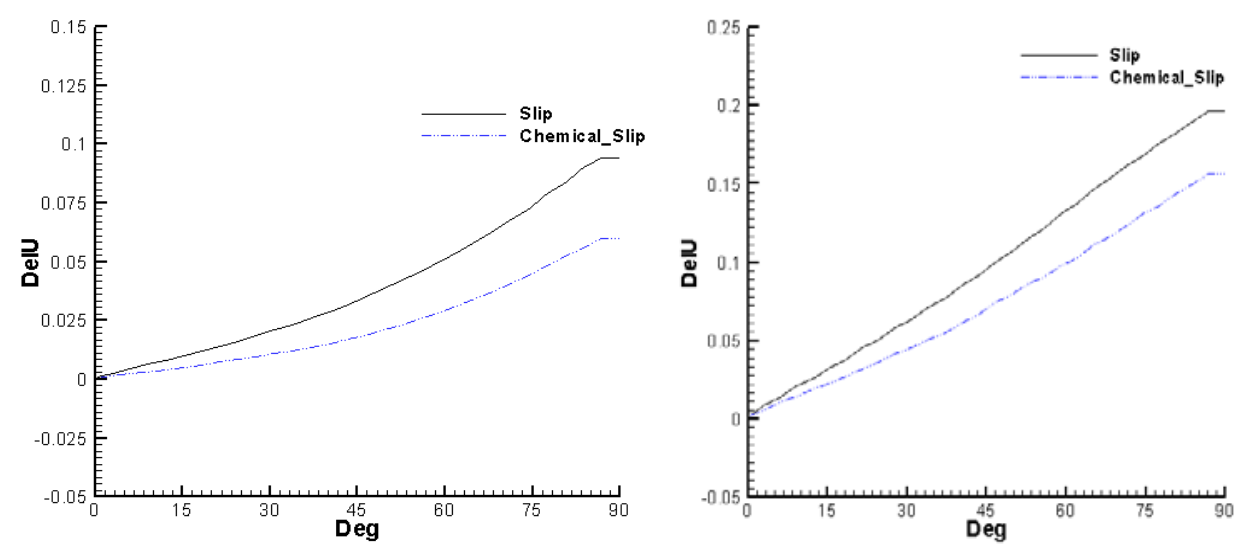

$80 \mathrm{~km}$

$90 \mathrm{~km}$

Fig.7. the comparison of slip velocity

Turn to surface slip velocity. The incensement value of surface velocity is shown on y-axis DelU, which is non-dimensional from incoming velocity. Results (Fig.7) come out that the chemical-equilibrium effect decrease DelU, and this trend is getting stronger with the increasing $\mathrm{Kn}$ number.

\section{Conclusions}

Through the research on high altitude of large Mach number chemical-equilibrium slip flow, we know that in hypersonic slip flow regime, chemical-equilibrium effects make the shock layer thinner, pressure higher and temperature lower behind the shock, the surface heat flux coefficient and slip velocity get smaller in which this trend becomes stronger with the rising Knudsen number.

\section{References}

[1] Tian Zhengyu. Numerical Investigation on Hypersonic MHD Control [D]. National University of Defense Technology, Changsha, 2008.

[2] Li Hua, Tian Zhengyu. Numerical Simulation on the Effects of Hypersonic Slip Flow [C]. Near Space CFD Seminar, Beijing, 2010.

[3] Yoon S, Jameson A. Lower-Upper Symmetric Gauss-Sediel Method for the Euler and Navier-Stokes Equations [J]. AIAA Journal, 1988, 26(9).

[4] Luo Kai, Pan Sha, Li Hua. Research on Computational Heat Flux Model in Hypersonic Slip Flow [C]. Dynamical Branch of Aerodynamic Academy in China, Chengdu, 2011.

[5] Tannehill J C, Mugge P H. Improved Curve Fits for the Thermodynamic Properties of Equilibrium Air Suitable for Numerical Computation Using Time-Dependent or Shock-Capturing Methods [R].Washington D.C. NASA CR-2470, 1974. 